\title{
The effect of APH treatment on surface bonding and osseointegration of Ti-6AI-7Nb implants: An in vitro and in vivo study
}

\author{
Thuy-Duong Thi Nguyen,, ${ }^{1}$ So-Hee Moon, ${ }^{2}$ Tae-Ju Oh, ${ }^{3}$ II-Song Park, ${ }^{1,4}$ Min-Ho Lee, ${ }^{1}$ \\ Tae-Sung Bae ${ }^{1}$ \\ ${ }^{1}$ Department of Dental Biomaterials and Institute of Oral Bioscience, Brain Korea 21 Plus Project, School of Dentistry, Chon- \\ buk National University, Jeonju 561-756, South Korea \\ ${ }^{2}$ Department of Periodontology, School of Dentistry, Chonbuk National University, Jeonju 561-712, South Korea \\ ${ }^{3}$ Department of Periodontics and Oral Medicine, School of Dentistry, University of Michigan, Ann Arbor, Michigan \\ ${ }^{4}$ Division of Advanced Materials Engineering, Chonbuk National University, Jeonju 561-756, South Korea
}

Received 22 October 2013; revised 13 April 2014; accepted 29 April 2014

Published online 27 June 2014 in Wiley Online Library (wileyonlinelibrary.com). DOI: 10.1002/jbm.b.33210

\begin{abstract}
This study investigated the effects of anodizationcyclic precalcification-heat (APH) treatment on the bonding ability of $\mathrm{Ca}-\mathrm{P}$ coating to the parent metal and osseointegration of Ti-6Al-7 Nb implants. Eighteen Ti-6Al-7Nb discs, 9 untreated and $9 \mathrm{APH}$-treated, were cultured with osteoblast cells in vitro, and the cellular differentiation ability was assayed at 1, 2, and 3 weeks. For in vivo testing, 28 Ti-6Al$7 \mathrm{Nb}$ implants (14 implants of each group) were inserted to rat tibias, and after each 4 and 6 weeks of implantation, bone bonding, and osseointegration were evaluated through removal torque and histological analysis. Osteoblastculturing showed twice as much of the alkaline phosphatase activity on the treated surface at 3 weeks than on the untreated surface $(p<0.05)$. The treated implants exhibited
\end{abstract}

higher removal torque values than the untreated ones (15.5 vs. $1.8 \mathrm{Ncm}$ at 4 weeks and 19.7 vs. $2.6 \mathrm{Ncm}$ at 6 weeks, $p<0.05)$. Moreover, the excellent bonding quality of coats was confirmed by the existence of cohesive fractures on the surface of removed APH implants (field emission scanning electron microscopy and histological observation). Within the limits of this study, it can be concluded that the APH treatment significantly enhanced osseointegration of the Ti-6Al$7 \mathrm{Nb}$ implant, with the stable bonding between the coating and the implant surface. (๑) 2014 Wiley Periodicals, Inc. J Biomed Mater Res Part B: Appl Biomater, 103B: 641-648, 2015.

Key Words: Ti-6Al-7Nb alloy, Ca-P coating, $\mathrm{TiO}_{2}$ nanotubes, delamination, osseointegration

How to cite this article: Thi Nguyen T-D, Moon S-H, Oh T-J, Park I-S, Lee M-H, Bae T-S. 2015. The effect of APH treatment on surface bonding and osseointegration of Ti-6Al-7Nb implants: An in vitro and in vivo study. $\mathrm{J}$ Biomed Mater Res Part B 2015:103B:641-648.

\section{INTRODUCTION}

Integration between the load-bearing implant surface and the living bone, osseointegration, is a prerequisite for implant success. The successful osseointegration begins with a biomechanical stability of the implant and a deposition of new bone along the surface. ${ }^{1}$ Modifying the geometry and/or the chemistry of the implant surface is still an area of interest in implant research. ${ }^{2-4}$

Hydroxyapatite (HA) coating has been one of the most common strategies for modifying implant surface., ${ }^{5,6}$ It was shown that a high concentration of calcium/phosphate favored tissue response by accelerating and enhancing the fixation to the bone without any soft tissue interactions. ${ }^{7-9}$ Calcium-phosphate (Ca-P) ceramics are considered to possess bioactive properties via a chemical bonding between the materials and bone. However, Ca-P ceramic-coated titanium-substrate has some disadvantages such as porous coating, residual stress at the substrate/coating interface, dramatic changes in the composition and crystalline property of the initial Ca-P powder. ${ }^{10,11}$ These can cause the surface coating to be delaminated at the implant-coating interface even if the coating is well-attached to the bone. $^{12,13}$ This phenomenon was recorded especially in the titanium implant coated by plasma-spraying-one of the methods that are frequently used in clinical settings. ${ }^{12-14} \mathrm{~A}$ clinical study of David et al. (1995) found out that the incidence of loosening increased progressively up to 55\% after 9 months of HA-coated implants loading with coat delamination. ${ }^{12}$ On the other hand, some literature described HA coating delamination as a significant theoretical problem. ${ }^{13,14}$ Debris of HA from coat loosening may activate macrophages, decrease local $\mathrm{pH}$, and induce bacterial colonization, resulting in bone loss. Thus, HA coating delamination could lead to clinical failure of the implant. ${ }^{13,14}$

Correspondence to: T.-S. Bae (bts@jbnu.ac.kr)

Contract grant sponsor: National Research Foundation of Korea (NRF), Ministry of Education, Science and Technology, Korea; contract grant numbers: 2010-0013251, 2012-012671 
In order to promote mechanical retention of Ca-P coating on the implant surface, the surface of implants can be roughened to provide anchorage for Ca-P particles. ${ }^{15,16}$ Anodization on implant surfaces with titanium or titanium alloys is often employed to form $\mathrm{TiO}_{2}$ nanotube layers, which increases surface roughness and enhances attachment of Ca-P particles to the implant surface. ${ }^{17-20}$ On the basis of this context, anodization-cyclic precalcification-heat (APH) treatment has been applied successfully on Ti-6Al-7Nb surface in our previous study. ${ }^{18}$ The nanosized Ca-P precipitates could penetrate not only between but also inside the nanotubes, thus forming interlocking Ca-P crystals within the tubes. ${ }^{18}$ Hence, a good adhesion of the coating to the substrate is expected. Moreover, the precalcification layer is quite thin (1-2 $\mu \mathrm{m})$ which makes it suitable to minimize potential problems of coat loosening. ${ }^{21}$ The proliferation activity of osteoblasts on Ti-6Al-7Nb surfaces has been enhanced by APH treatment during 1-week culturing. ${ }^{18}$ However, it has not been demonstrated whether this initial biological behavior would be correlated with their in vivo performance. Therefore, this study was conducted to investigate effects of the APH treatment on surface bonding and osseointegration of nanotubular Ti-6Al-7 Nb implants in vitro and in vivo.

\section{MATERIALS AND METHODS}

Preparation of cyclic precalcified nanotubular samples (APH treatment)

APH samples were prepared as previously described. ${ }^{18}$ Ti6Al-7Nb alloy samples (T-Alloy Tough, GC Corp., Tokyo, Japan) were anodized with glycerol-distilled water (DW)$\mathrm{NH}_{4} \mathrm{~F}(79,20$, and $1 \mathrm{wt} \%$, respectively) electrolyte to form $\mathrm{TiO}_{2}$ nanotubes. Cyclic precalcification was subsequently performed by exposing the nanotube-formed specimens sequentially in $80^{\circ} \mathrm{C}-0.05 \mathrm{M} \mathrm{NaH_{2 }} \mathrm{PO}_{4}$ and $100^{\circ} \mathrm{C}$-saturated $\mathrm{Ca}(\mathrm{OH})_{2}$ solutions for $1 \mathrm{~min}$ each turn. This process was repeated for 20 cycles and finished by heat treatment at $500^{\circ} \mathrm{C}$ for $2 \mathrm{~h}$.

\section{In vitro test}

Eighteen Ti-6Al-7Nb discs measuring $16 \mathrm{~mm}$ in diameter and $1 \mathrm{~mm}$ in thickness were prepared and then polished with up to \#1000 grit SiC paper, followed by a removal of old oxide layer in $\mathrm{HNO}_{3}: \mathrm{HF}: \mathrm{H}_{2} \mathrm{O}(12: 7: 81$ vol \%, respectively) solution for $20 \mathrm{~s}$. Nine samples were submitted to above APH treatment and $37.5^{\circ} \mathrm{C}$ simulated body fluid (SBF)-immersed for 2 days (APH group) and the other nine untreated samples were used as control group (UT group).

Mouse osteoblast cells (MC3T3-E1) were subcultured over the prepared samples with an initial density of $10^{4} / \mathrm{mL}$ in order to evaluate their differentiation behavior. $\alpha$-MEM (Gibco, USA) containing 10\% fetal bovine (Gibco, USA), 500 unit/mL of penicillin (Gibco, USA) and 500 unit/mL of streptomycin (Gibco, USA) was used as the growth culture medium. The medium was changed every 2 days, and the cultures were maintained at $37^{\circ} \mathrm{C}$ in a humidified 5 vol \% $\mathrm{CO}_{2}$ atmosphere. At 1, 2, and 3 weeks after incubation, the differentiation of osteoblast cells was evaluated through the expression of alkaline phosphatase (ALP) activity using the Sensolyte ${ }^{\circledR}$ pNPP ALP Assay Kit (AnaSpec, CA). The samples were washed twice with $1 \mathrm{X}$ buffer (AnaSpec, CA) to remove culture medium. Then, each samples were added $200 \mu \mathrm{L}$ of $0.2 \%$ Triton X-100 (AnaSpec, CA) to lyse the cells. The lysate was collected in a microcentrifuge tube using a cell scraper, incubated at $4^{\circ} \mathrm{C}$ for $10 \mathrm{~min}$ under agitation and centrifuged at $2500 \mathrm{rpm}$ for $10 \mathrm{~min}$ at $4^{\circ} \mathrm{C}$. A $50 \mu \mathrm{L}$ of supernatant was mixed with $50 \mu \mathrm{L}$ of p-nitrophenyl phosphate (pNPP) (AnaSpec, CA). The mixture was incubated at $37.5^{\circ} \mathrm{C}$ for $30 \mathrm{~min}$ and stopped by adding $50 \mu \mathrm{L}$ of $\mathrm{NaOH}$ solution (AnaSpec, CA) in each well. The final solution showed a yellow-colored product due to conversion of $\mathrm{p}$ nitrophenyl phosphatase to p-nitrophenol and was measured spectrophotometrically at $405 \mathrm{~nm}$ (microplate reader, Molecular Devices, CA). The ALP activity was read off from a standard curve obtained with ALP concentrations of $0-$ $200 \mathrm{ng} / \mathrm{mL}$. This test was completed with triplicate samples.

\section{In vivo tests}

After obtaining a fresh oxide layer by above etching step, we divided 28 cylindrical implants $(2-\mathrm{mm}$ diameter $\times 4$ mm length) manufactured from Ti-6Al-7Nb alloy (MEGAGEN, Daegu, Korea) into the untreated group (UT) with machine surface and the APH-treated group (APH) for animal experiments. Prior to in vivo tests, implant surfaces were analyzed by using field emission scanning electron microscopy (FE-SEM in KBSI Jeonju, S-4700, Hitachi, Tokyo, Japan) equipped with energy dispersive X-ray analysis (EDS, Bruker, Germany).

This in vivo study was conducted in compliance with the Declaration of the Helsinki, and it was approved by the Institutional Animal Care and Use Committee of the Chonbuk National University Laboratory Animal Center.

Fourteen male Sprague Dawley rats (9-week-old), weighing $\sim 300 \mathrm{~g}$, were allowed to acclimate about 1 week prior to study. The rats were deeply anesthetized via the intraperitoneal injection of $50 \mathrm{mg} / \mathrm{kg}$ of tiletamine plus zolazepam (Zoletil 50, Virbac Laboratories, Carros, France) and $15 \mathrm{mg} /$ $\mathrm{kg}$ of xylazine hydrochloride (Rompun, Leuverkeusen, Germany). Additional local anesthesia was given on the surgical site (tibia) using $1 \mathrm{~mL}$ of $2 \%$ lidocaine with epinephrine (1 : 100,000). The surgical area was shaved and disinfected with an iodine scrub. The implantation process was described in Figure 1(A-D). A 1-cm incision was made on the tibia [Figure 1(A)]. After flap reflection, a $1.8 \mathrm{~mm}$ pilot bur (H1.316018, Komet, Germany) was used to score a hole on the cortical bone, followed by preparation of an implant bed in the medial region of tibia diaphysis using a $2.0 \mathrm{~mm}$ guide drill (Neoplant Unity Surgical Kit, Neobiotech, Korea) [Figure 1(B)]. The operation was done under a copious saline irrigation, at a rotary speed of $\leq 350 \mathrm{rpm}$. The implants were press-fit in their designated positions using gentle tapping [Figure 1(C)]. For each rat, the UT implant was placed on one tibia and the APH implant was placed on the opposite side. A 4/0 suture silk (4/0 Black silk, Ailee Co., Busan, Korea) was used to approximate the surgical 

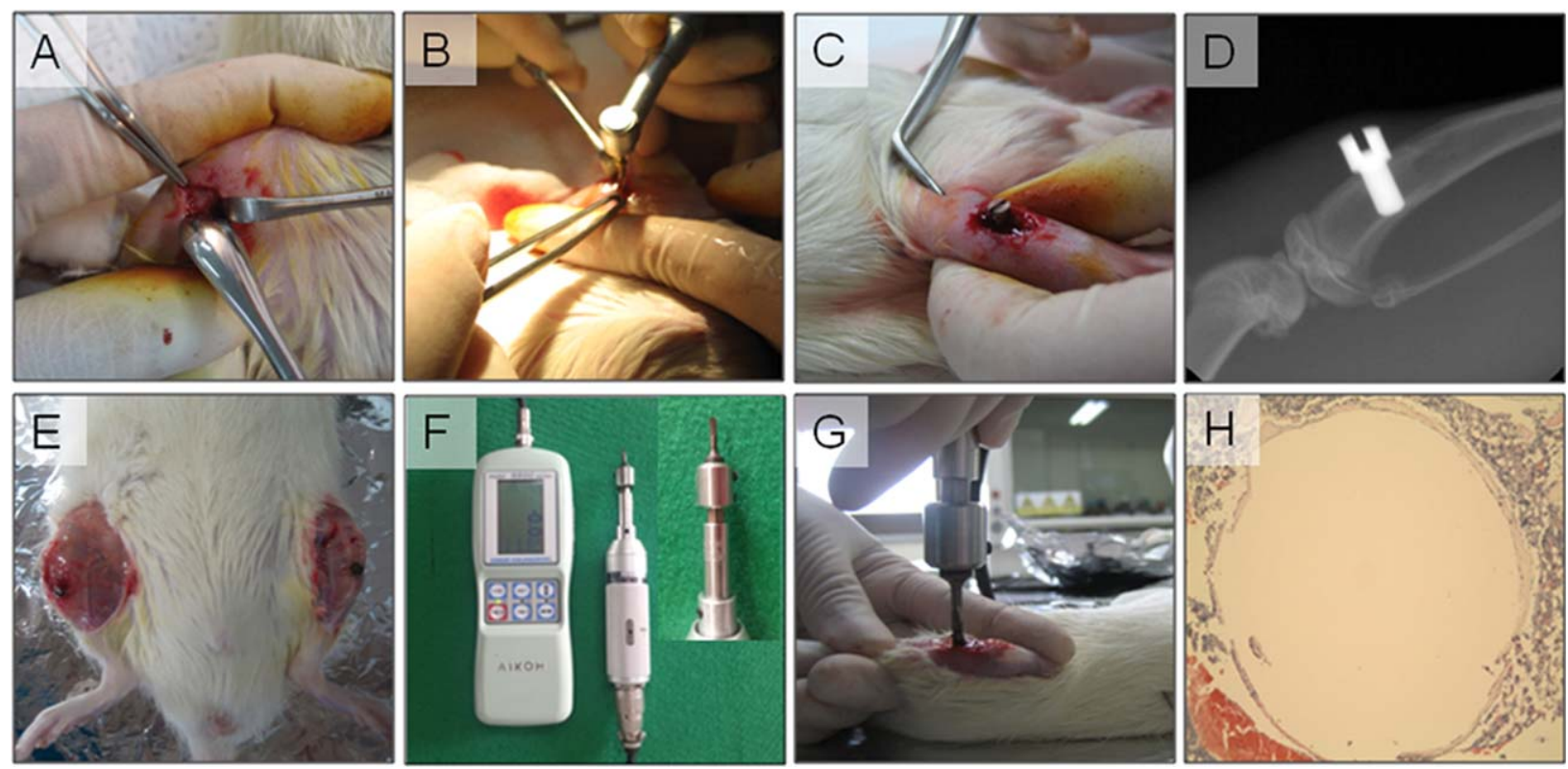

FIGURE 1. Implantation procedure (A-D) and removal torque measurement in rat tibia (E-H). A, Bone exposure; B, Implant bed preparation; C, Implant placement; D, Implant position on X-ray image; E, Implantation area exposure after 4-6 weeks; $F$, Digital torque gauge with a detailed drawing of $0.1 \mathrm{Ncm}$; G, remove torque measurement; $\mathrm{H}$, Cross section of implant area after torque removal measurement (H\&E staining, $\times 50$ ). [Color figure can be viewed in the online issue, which is available at wileyonlinelibrary.com.]

wound. Antibiotics (300 $\mu \mathrm{L} / \mathrm{kg})$ were administered subcutaneously (Amikacin, Samu media, Korea) at 0, 24, and 48 h, postoperatively. This was followed by an intraoral radiography (Kodak 2200, Trophy, France) of the rat tibia to evaluate implant position at $60 \mathrm{kV}$ and $7 \mathrm{~mA}$ for $0.125 \mathrm{~s}$ [Figure 1(D)]. At 4 and 6 weeks after the implantation, seven rats from each were sacrificed by thiopental overdose (Thiopental sodium, ChoongWaePharma, Seoul, Korea).

\section{Removal torque analysis}

Removal torque value (RTV) measurement, which was conducted in 5 rats each at 4 and 6 weeks, is depicted in Figure $1(\mathrm{E}-\mathrm{H})$. The implant sites in the rat tibias were surgically exposed via a sharp dissection and the overgrowing bone and soft tissues were carefully removed [Figure 1(E)]. Removal torque tests were performed on two tibias of a rat using a digital torque gauge (9810P, Aikoh Engineering, Osaka, Japan) with a detailed drawing of $0.1 \mathrm{Ncm}$ [Figure 1(F)]. After stabilizing the tibia, an implant removal mount was securely fastened, engaging the external hex that was connected to the torque gauge with the insertion device .The gauge was aligned with the implant axis and torque was increased incrementally by slowly rotating the gauge in order to rupture the bone-implant interface [Figure 1(G)]. The RTV was measured by a single examiner and recorded in $\mathrm{Ncm}$ for each group.

After removal torque test, the implants were collected for surface observation by FE-SEM (FE-SEM in KBSI Jeonju, S-4700, Hitachi, Tokyo, Japan) and EDS (Bruker, Germany). Implant-removed tibia blocks were harvested from 5 rats after the removal torque test at each time point ( 4 and 6 weeks) and fixed in $10 \%$ formalin solution for 2 days. The blocks were decalcified, then cut in $1 \mathrm{~cm}$ in length and dehydrated in a series of increasing concentrations of ethanol $(70 \%, 80 \%, 90 \%, 95 \%, 100 \%)$ before embedding in paraffin. Paraffin embedded block were sectioned as perpendicular to the longitudinal axis of the implant at a thickness of $5 \mu \mathrm{m}$ by using a rotary microtome (Leica RM 2235, Wetzlar, Germany) and mounted on glass slides. The sections were stained with a hematoxylin and eosin (H\&E) dye and then inspected under an optical microscope (Leica DM $2500 \mathrm{M}$, Germany) to assess the histology of breakage interface [Figure $1(\mathrm{H})]$.

\section{Histological observation}

The bone-implant interface was histologically observed at 4 and 6 weeks postoperatively. Implant-installed tibial blocks from the remaining two rats at each time point (4 and 6 weeks) were fixed in 10\% formalin solution for 2 days, stained in Villanueva bone stain solution for 3 days, and dehydrated in a series of increasing concentrations of alcohol before embedding in methylmetacrylate. Afterwards, the blocks were sectioned and ground as parallel to the longitudinal axis of the implants to a thickness of about 10-40 $\mu \mathrm{m}$. Then, the bone-implant interface was examined by an optical microscope (Leica DM 2500M, Germany).

\section{Statistical analysis}

The ALP assay results and RTV were expressed as mean \pm SD (SD: standard deviation). Statistical analysis was performed using one-way analysis of variance (ANOVA) with Tukey tests. A $p$-value of $<0.05$ was considered statistically significant. All statistical analyses were carried out using 

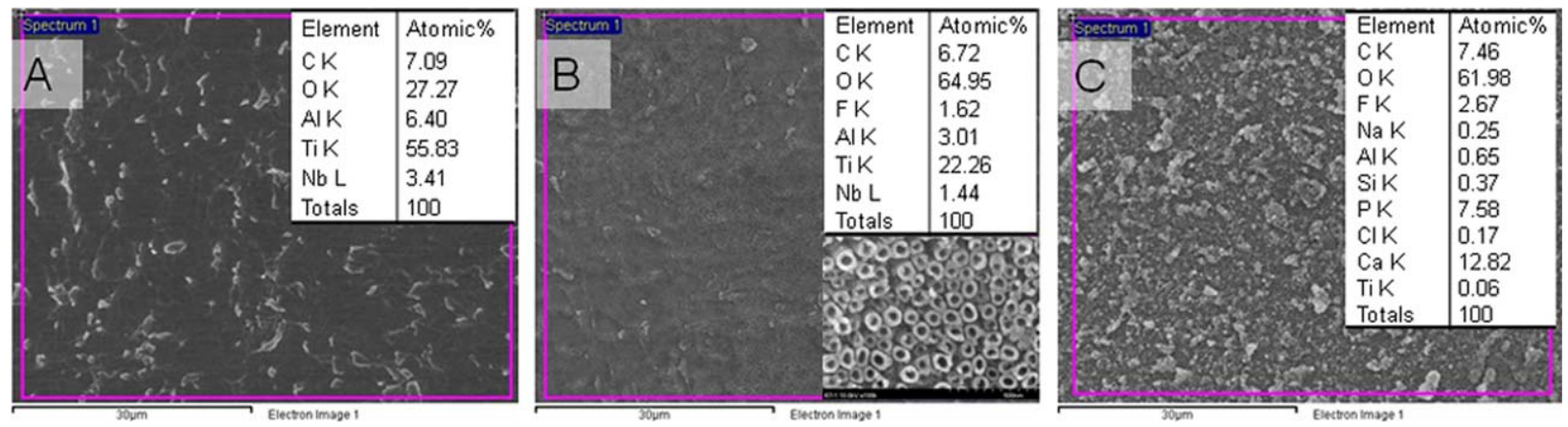

FIGURE 2. FE-SEM images $(\times 3.000)$ and EDS analysis results of the surfaces: $A$, Machine turned surface (UT); $B$, Anodized surface with nanotube structure (inset image, $\times 100.000)$; $C$, Cyclic precalcified nanotubular surface $(\mathrm{APH})$. [Color figure can be viewed in the online issue, which is available at wileyonlinelibrary.com.]

computer software, Statistical Package for the Social Sciences (SPSS ver12.0, SPSS, Chicago, IL).

\section{RESULTS}

Figure 2 illustrates surface morphology and chemical composition of Ti-6Al-7Nb alloy implant through SEM-EDS examination after cyclic precalcified nanotubular treatment. The figure shows the untreated surface [Figure 2(A)], nanotubular structures formed after the anodization [Figure 2(B)], and a rough Ca-P layer completely covering the $\mathrm{TiO}_{2}$ substrate after the cyclic precalcification [Figure 2(C)]. Upon analyzing EDS of these surfaces, detected oxygen level increased in atomic percentage after anodization treatment, which means that a thicker $\mathrm{TiO}_{2}$ layer has been formed on sample surfaces. After precalcification, the mineral deposits on APH surface contained calcium and phosphate with the $\mathrm{Ca} / \mathrm{P}$ ratio of 1.69 , same to $\mathrm{Ca} / \mathrm{P}$ ratio of hydroxyapatite.

\section{Osteoblasts culture}

As seen from Figure 3, the ALP activity of seeded osteoblasts on both two surfaces was increased from week 1 to

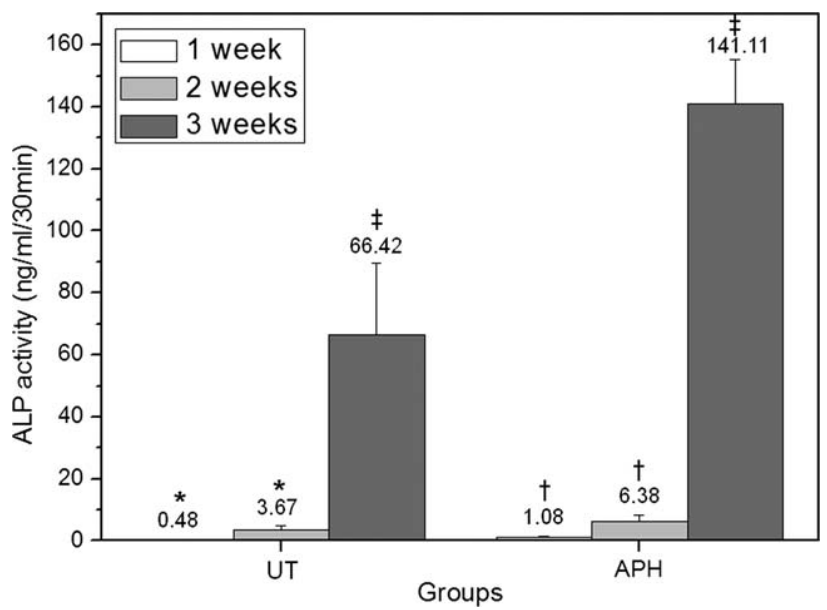

FIGURE 3. Differentiation (alkaline phosphate activity) of osteoblastic cells cultured on untreated (UT) and treated surfaces (APH) after 1, 2, and 3 weeks. All data are reported as mean \pm standard deviation. ${ }^{*} p<0.05$ indicates a statistically significant difference to 3 week-UT. $+p<0.05$ indicates a statistically significant difference to 3 -week-APH. $\neq p<0.05$ indicates a statistically significant difference between UT and $\mathrm{APH}$. week 3 , dramatically at third week $(p<0.05)$. During the first 2 weeks, the osteoblastic differentiation of the APH group showed a comparable activity to the UT groups. However, a significantly accelerated activity in the APH group was recorded at 3 weeks which over twenty-fold higher than that at 2 weeks and doubled the result of the UT group at the same assessed time $(p<0.05)$.

\section{Removal torque test}

Table I presents the removal torque measured at 4 and 6 weeks after placing the dental implants. The torque measurements revealed values under $5 \mathrm{Ncm}$ on the control group at both periods. In APH groups, the mean resistance to torque removal increased from $15.5 \pm 3.3 \mathrm{Ncm}$ to $19.7 \pm 2.2$ $\mathrm{Ncm}$ at 4- to 6-week intervals, exhibiting a significant higher value as compared with controls at both time points with ratio of 7.6 and 8.6, respectively $(p<0.05)$.

After implant removal for removal torque analysis, the remaining tissues as well as the extracted implant surfaces were histological observed. The surrounding area of UT implant showed only connective tissue at 4 weeks, but new bone remnants were observed at 6 weeks [Figure 4(A,B)]. In the APH group, at both 4 and 6 weeks, new bones were detected on surrounding tissue with darker stains and more breakages [Figure 4(C,D)]. FE-SEM and EDS analysis [Figure $4(\mathrm{E}, \mathrm{F})]$ of UT implant surfaces revealed few of attached tissues with $\mathrm{Ca} / \mathrm{P}$ ratio around 1.5 but in other areas where $\mathrm{Ca}$ and $\mathrm{P}$ were unidentified. Meanwhile, most of APH implant surfaces were covered by a rather thick layer of bone tissue where the $\mathrm{Ca} / \mathrm{P}$ ratio was around 1.67 [Figure $4(G, H)]$. Therefore, interface fractures between new bone and implant in the UT group and cohesive fractures within newly formed bone in the APH group were observed.

TABLE I. Removal Torque Values at 4 and 6 Weeks ( $n=5$ Per Time Point)

\begin{tabular}{lcc}
\hline Removal Torque & UT Group & APH Group \\
\hline 4 weeks $(\mathrm{Ncm})$ & $1.8 \pm 1.1\left(^{*}\right)$ & $15.5 \pm 3.2\left(^{*}\right)$ \\
6 weeks $(\mathrm{Ncm})$ & $2.6 \pm 0.6(\dagger)$ & $19.7 \pm 2.2(\dagger)$ \\
\hline
\end{tabular}

${ }^{*}, \dagger$ indicates statistically significant differences between the UT group and the APH group at 4 and 6 weeks, respectively $(p<0.05)$. 

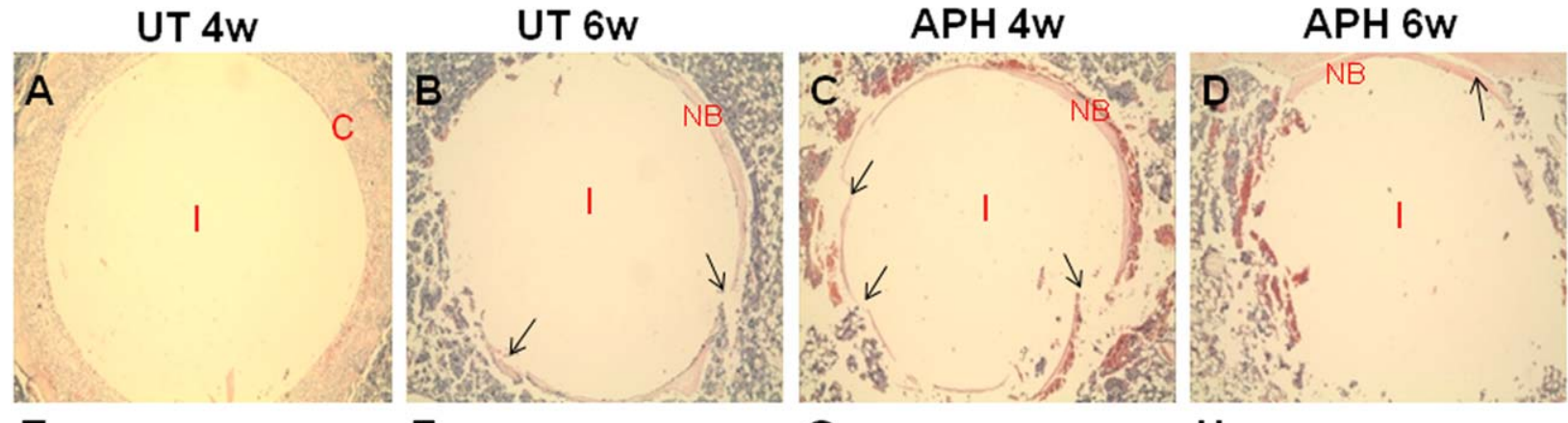

E

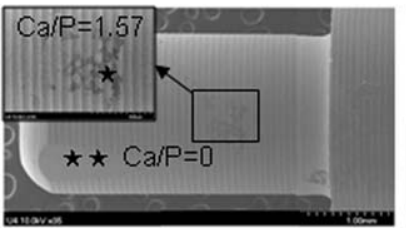

$\mathbf{F}$

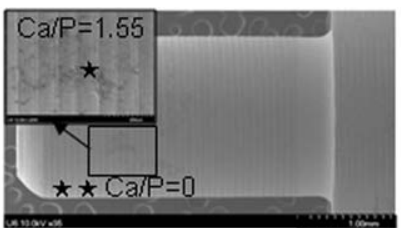

G

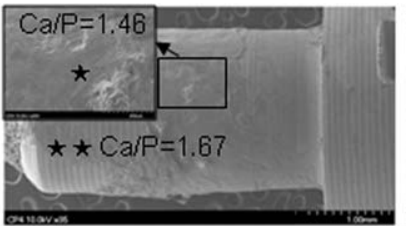

$\mathrm{H}$

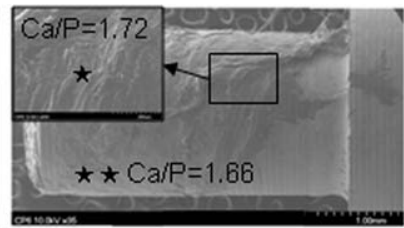

FIGURE 4. Histological observation after implant removal. A-D: Cross section of implant site (H\&E staining, $\times 50)$. E-H, Implant surface (FE-SEM, $\times 35)$, the onsets are the high magnification $(\times 200)$ of implant surface. I, implant area; C, connective tissue; NB, new bone tissue, arrows: new bone fractures $\star, \star \star \star$ : the EDS checked points. [Color figure can be viewed in the online issue, which is available at wileyonlinelibrary.com.]

\section{Histological observation of bone-implant interfaces}

The implant-installed tibial blocks were observed by the optical microscope for bone-implant interface examinations (Figures 5 and 6). The surface of UT implants at 4 weeks did not demonstrate the contact bone formation, some bone clusters were observed along implant axis [Figure 5(A)]. The gap between implant surface and these clusters were about $20 \mu \mathrm{m}$. Meanwhile, there was a thin layer of newly formed bone attaching on APH surface at the same period [Figure 5(B)]. That is suggestive that osseointegration is taking place between bone and implant surfaces in the APH group. At 6 weeks, both groups showed a bone adhesion without gaps along the implant axis, indicating a contact osseointegration [Figure 5(C,D)]. However, the bone layer covering the UT surface is thinner, lightly stained as compared with that on the APH surface. Figure 6 shows higher magnifications of bone-implant interface in the APH groups. At 4 weeks, de novo bone formed as an extracellular layer with the osteoblasts and osteocytes [Figure 6(A)]. Meanwhile, at 6 weeks, remodeling areas with existence of osteoclasts and osteoblasts inside a new bone layer indicate that active and mature bones were formed on modified surface [Figure 6(B)]. Thus, sites receiving implants coated with Ca$\mathrm{P}$ exhibited a mature bone formation at 6 weeks.

\section{DISCUSSION}

For this study, Ti-6Al-7Nb implants underwent a cyclic precalcified nanotubular (APH) treatment in order to form a
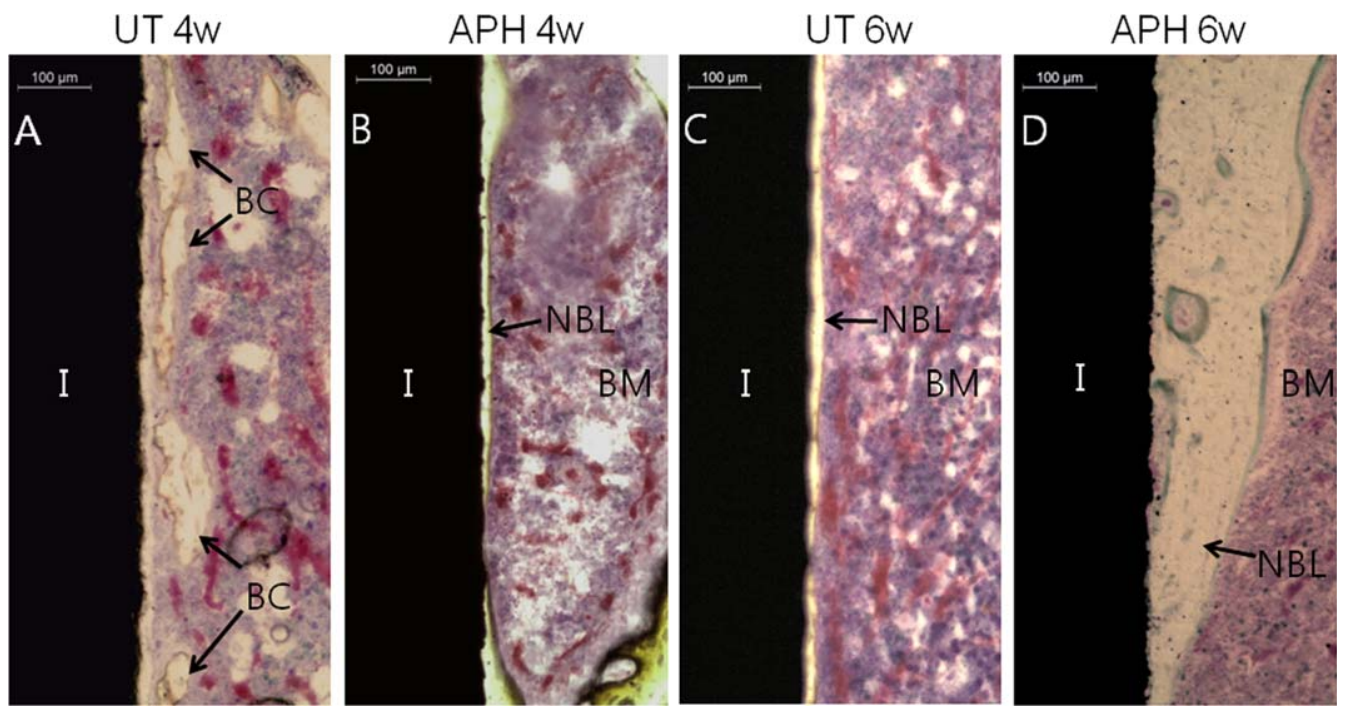

FIGURE 5. Histology of bone-implant interfaces (Villanueva bone staining, $\times 100$ ) in the UT group at 4 weeks (A) and 6 weeks (B) and the APH group at 4 weeks (C) and 6 weeks (D). I, implant; BM, bone marrow; BC, bone clusters; NBL, new bone layer. [Color figure can be viewed in the online issue, which is available at wileyonlinelibrary.com.] 

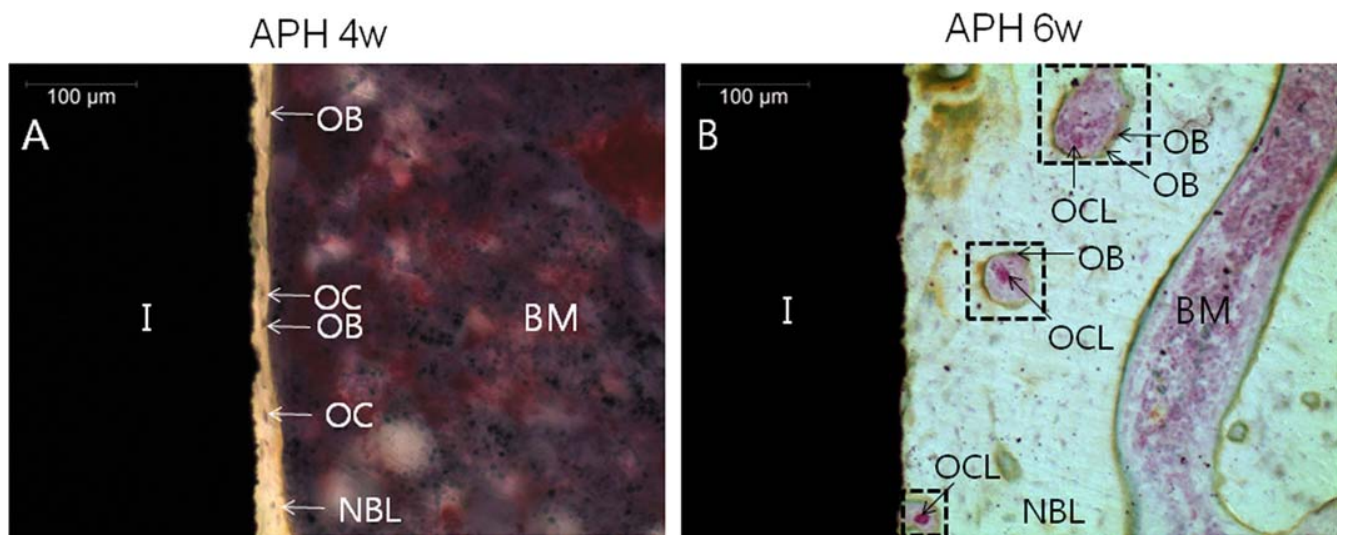

FIGURE 6. High magnification $(\times 200)$ from the surface of APH implant at 4 weeks $(\mathrm{A})$ showed de novo bone layer, and at 6 weeks $(\mathrm{B})$ showed remodeling areas (dashed squares). I, implant; BM, bone marrow; NBL, new bone layer; OB, osteoblast; OC, osteocyte; OCL, osteoclast. [Color figure can be viewed in the online issue, which is available at wileyonlinelibrary.com.]

biocompatible coating as mentioned in our previous study. ${ }^{18}$ Afterward, the degree of osseointegration of these modified implants were accessed in vitro and in vivo.

The APH treated Ti-6Al-7Nb substrate has exhibited a bio affinity for osteoblastic cells in our previous study. ${ }^{18}$ MTT assay showed that modified surface could provide a favorable rough and porous surface for MC3T3-E1 attachment and growth compare to smooth surface of UT. However, a slight decrease in proliferation rate on APH treated samples was recorded at the 7th day, proposing an early differentiation potential. ${ }^{18}$ Therefore, the activity of alkaline phosphatase, an enzyme that is involved in the osteoblastic differentiation and initial mineral extracellular matrix (ECM) formation, were evaluated from 1 to 3 weeks in this study. $^{22}$

The results showed significantly increased ALP activity on the APH surfaces than on the UT surfaces at 3 weeks. This phenomenon might be attributed to the change in surface morphology, roughness and chemical composition by precalcification treatment. ${ }^{3}$ The rough surfaces with the Ca$\mathrm{P}$ nanoparticles have been reported to enhance the attachment and differentiation of osteoblast, thus stimulating early bone formation better than unmodified surfaces. ${ }^{23-25}$ Besides that, the bioactivity property of APH coating, which was reported in the previous study, also might have an influence on the ECM formation increase. ${ }^{18,26}$ According to $\mathrm{Gu}$ et al.'s study, HA-deposited nanotube surface showed an improvement of both earlier osteogenic differentiation and matrix mineralization compared to plain nanotubes. ${ }^{26}$ It is believed that during the early healing phase, APH coating dissolves partly in the body fluid, along with precipitation of a biological Ca-P layer on the nanotubes. ${ }^{26-28}$ Subsequently, ECM-forming organic compounds would be incorporated into this newly formed layer, followed by colonization of osteoprogenitor cells, such as osteoblasts and osteoclasts. ${ }^{27,29}$ As a result, ECM formation would be promoted in a short period of time.

In addition, the results also represented the dramatic increase of ALP enzyme in both two groups at 3 weeks, which implied that the ECM formation might be induced strongly at this period. Thus the bone formation would happen afterwards. Therefore, a 4 -week period is desirable to obtain the intimate bone contact over the whole length of the implant surface. ${ }^{27}$ Different time intervals of the mechanical testing of implants are discussed in the literature: 2 weeks, ${ }^{30} 4$ weeks, ${ }^{31} 5$ weeks, ${ }^{32}$ and 9 weeks. $^{33}$ In this study, 4 and 6 weeks were selected as time points to assess the dynamic process of the formation of the bone/ implant interface in vivo.

Removal test is a dynamic one that considers the threedimension relationship between implant and bone, showing the strength of this attachment. ${ }^{34}$ During both assessment periods, higher friction forces between the implant and surrounding bone were well demonstrated in the treated group as compared with the UT group. This suggests a higher degree of osseointegration existed, which is in agreement with other reports that Ca-P coated titanium-implants displayed a higher mechanical fixation than uncoated ones. ${ }^{35}$ After biomechanical test, the removed implant surfaces and the fractured tissue were examined. Both at 4 and 6 weeks, FE-SEM observation in the APH group showed a high degree of bone contact with the implants, indicating that more bone had formed in the areas adjacent to the implant with a high bonding. In addition, bone fragments were also observed in surrounding tissue, concluding that fractures had happened between bone tissue layers. That is, the cohesive fractures existed. Meanwhile, in the UT group, at 4 weeks, connective tissue was detected around implant areas rather than the bone tissue. At 6 weeks, bone tissue was identified around the implant site though no trace of bone was found in the removed implant. This suggests that new bone was formed with a weak bond strength, which caused fracture at the bone-implant interface while exerting friction forces.

The current study demonstrated that the Ca-P coating had a good bonding strength with the Ti-6Al-7Nb substrate. This can be proposed as a better method to diminish coat delamination compared with plasma spraying method. Plasma sprayed Ca-P coated implants have been the most widely used in implant dentistry. However, the delamination 
or interface fracture has been highlighted as a major concern for the failure of these implants. ${ }^{12-14}$ This is because nonuniform coats with a thickness of 50-200 $\mu \mathrm{m}$ are created as well as poor adhesion between the coat and the underlying implant. ${ }^{14,36}$ It can therefore be regarded that our methods are useful in the creation of coating with a uniform thickness of 1-2 $\mu \mathrm{m}$, as it can cause positive long-term clinical results of implant by reducing the debonding or loosening of the surface lamination during the operation process. Recently, Ca-P coatings, which are produced using a sputtering process - an alternative to plasma spraying-have been developed. ${ }^{6}$ In a comparative study of Mohseni et al., the sputtering method can form a uniform, dense and thin (0.5-3 $\mu \mathrm{m})$ coating on flat substrates with highest adhesion strength respect to other methods. ${ }^{13}$ Ueda et al. investigated the effects of the sputtering method compared to uncoated implants with rabbit femur. ${ }^{37}$ Comparing with our RTV results, RTV ratio of the APH implant/the UT implants is higher than that of the sputtered implants and uncoated implants (8.6 and 1.3, respectively) after a period of 4 weeks. ${ }^{37}$ Because of different studying conditions, a direct comparison between the methods is impossible to carry on, however, this fact indicates that APH coating strongly enhanced biomechanical property of bare substrate. Ascribing for this, underlying nanotubes played an important role. It is apparent that mechanical stability of the Ca-P coating also requires a rough titanium surface to form a good connection with metallic substrate like nanotubes which formed directly from $\mathrm{Ti}$ base. ${ }^{16,20,38}$ Cyclic-coating procedures gradually deposited Ca-P particles inside the nanotubes which may result in the growth of apatite into and on the tubes, forming a strong link between substrate and upper Ca-P layer. ${ }^{18}$

In this study, the interface of bone-implant was histologically observed at the same two time points, 4 and 6 weeks, to investigate the patterns of osseointegration in test and control groups. The results revealed a notable difference between the two groups. Contact osteogenesis was established between the new bone and APH implant at 4 weeks, and replaced with more mature bone tissue at 6 weeks. Whereas, a gap was detected between the UT surface and bone clusters at 4 weeks, followed by appositional bone growth from the parent bone layer, at 6 weeks, as a thin layer of woven bone. The osseointegration has been defined as a histological concept with direct contact of bone to implant at the resolution level of the optical microscope. ${ }^{39}$ Therefore, it can be stated that osseointegration was taking place at 4 and 6 weeks on the APH and UT implant, respectively. Moreover, the maturation of the new bone layer on the APH implant was induced through the transformation from de novo bone at 4 weeks to lamellae bone with existence of bone reformation or remodeling at 6 weeks. This de novo bone adhesion to the implant surface during the early stage, may lead to a desirable osseointegration. ${ }^{2}$ This enhancement in osseointegration has been reported in many past studies, which said that a high concentration of Ca-P coating on a titanium surface produces a favorable tissue response by accelerating the fixation to the bone without any soft tissue interactions. ${ }^{7-9}$
It is possible that the positive effects of nano structures seen at a cellular level disappear when screw-shaped implants, with a pronounced microroughness, are used. It will cause hardship to detect the effects in in vivo. In this study, we therefore used an experimental model of the cylindrical implant without screws to rule out the effects of screw-in and screw-out force, as well as to perform histological examinations of the remnant tissue around implant area. However, with this implant model, the ability of press fit of screws/ threads in bone is also eliminated. Therefore, the RTV in this study is not as high as in other studies where screw-shaped implants with various surface modifications were used. ${ }^{39,40}$ Additional studies, including other surface modifications with a same design, are required to clarify the effectiveness of this APH coating on biomechanical properties.

Because our surface modifications were performed in an aqueous condition, it is plausible that the modifications on implants with complex shapes and multiple threads would also be applicable. However, the integrity of this coating might be damaged by the high shear forces that are generated during the insertion of screw-like implants. If there are any chances of coatings with an osteoinductive agent such as bone morphogenic protein-2 (BMP-2), the fragments of the coating formed during implant insertion will retain their depots in surrounding sites, and their osteoinductive potential is still effective. ${ }^{35}$ Further studies are therefore warranted to examine the effects of the combination of these agents with osteoconductive coating.

In summary, the overall qualitative evaluation in this study revealed that the APH surface had a positive effect on the early healing and mechanical fixation of implants. Additional studies, including a comparison of the above treatments with the groups of nanotubular layers without the precalcification treatment, and HA-coating without nanotubular layers, would be required to clarify whether the result was due to a roughness from the nanotubes or due to a chemical composition of Ca-P precipitates. And a possible limitation of this study was that histomorphometric analysis was not conducted for quantity evaluation of bone-implant contact.

\section{REFERENCES}

1. Novaes AB Jr, Souza SLSd, Barros RRMd, Pereira KKY, lezzi G, Piattelli A. Influence of implant surfaces on osseointegration. Brazilian Dental J 2010;21:471-481.

2. Elias CN, Oshida Y, Lima JHC, Muller CA. Relationship between surface properties (roughness, wettability and morphology) of titanium and dental implant removal torque. J Mech Behav Biomed Mater 2008;1:234-242.

3. Habibovic P, Li J, van der Valk CM, Meijer G, Layrolle P, van Blitterswijk CA, de Groot K. Biological performance of uncoated and octacalcium phosphate-coated Ti6AI4V. Biomaterials 2005;26: 23-36.

4. Strnad J, Strnad Z, Šesták J, Urban K, Povýšil C. Bio-activated titanium surface utilizable for mimetic bone implantation in dentistry.III. Surface characteristics and bone-implant contact formation. J Phys Chem Solids 2007;68(5-6):841-845.

5. Junker R, Dimakis A, Thoneick M, Jansen JA. Effects of implant surface coatings and composition on bone integration: A systematic review. Clin Oral Implants Res 2009;20:185-206.

6. Yang $\mathrm{Y}$, Kim K-H, Ong JL. A review on calcium phosphate coatings produced using a sputtering process-An alternative to plasma spraying. Biomaterials 2005;26:327-337. 
7. Barrère $F$, van der Valk CM, Meijer G, Dalmeijer RAJ, de Groot $K$, Layrolle $\mathrm{P}$. Osteointegration of biomimetic apatite coating applied onto dense and porous metal implants in femurs of goats. J Biomed Mater Res B Appl Biomater 2003;67:655-665.

8. Geurs NC, Jeffcoat RL, McGlumphy EA, Reddy MS, Jeffcoat MK. Influence of implant geometry and surface characteristics on progressive osseointegration. Int J Oral Maxillofacial Implants 2002; 17:811-815.

9. Morris HF, Ochi S, Spray JR, Olson JW. Periodontal-type measurements associated with hydroxyapatite-coated and non-HAcoated implants: Uncovering to 36 months. Annals Periodontol 2000;5:56-67.

10. Giavaresi G, Fini M, Cigada A, Chiesa R, Rondelli G, Rimondini L, Torricelli P, Aldini NN, Giardino R. Mechanical and histomorphometric evaluations of titanium implants with different surface treatments inserted in sheep cortical bone. Biomaterials 2003;24:1583-1594.

11. Lee JJ, Rouhfar L, Beirne OR. Survival of hydroxyapatite-coated implants: A meta-analytic review. J Oral Maxillofacial Surg 2000; 58:1372-1379.

12. Dávid A, Eitenmüller J, Muhr G, Pommer A, Bär HF, Ostermann PAW, Schildhauer TA. Mechanical and histological evaluation of hydroxyapatite-coated, titanium-coated and grit-blasted surfaces under weight-bearing conditions. Arch Orthopaedic Trauma Surg 1995; 114:112-118.

13. Mohseni E, Zalnezhad E, Bushroa AR. Comparative investigation on the adhesion of hydroxyapatite coating on Ti-6Al-4V implant: A review paper. Int J Adhesion Adhesives 2014;48:238-257.

14. Sun L, Berndt CC, Gross KA, Kucuk A. Material fundamentals and clinical performance of plasma-sprayed hydroxyapatite coatings: A review. J Biomed Mater Res 2001;58:570-592.

15. Chen X, Li Y, Hodgson PD, Wen Ce. Microstructures and bond strengths of the calcium phosphate coatings formed on titanium from different simulated body fluids. Mater Sci Eng C 2009;29:165-171.

16. Svehla M, Morberg P, Bruce W, Zicat B, Walsh WR. The effect of substrate roughness and hydroxyapatite coating thickness on implant shear strength. J Arthroplasty 2002;17:304-311.

17. Mîndroiu M, Pirvu C, Ion R, Demetrescu I. Comparing performance of nanoarchitectures fabricated by Ti6Al7 Nb anodizing in two kinds of electrolytes. Electrochimica Acta 2010;56:193-202.

18. Nguyen T-DT, Park I-S, Lee M-H, Bae T-S. Enhanced biocompatibility of a pre-calcified nanotubular TiO2 layer on Ti-6Al-7Nb alloy. Surf Coat Technol 2013;236:127-134.

19. Kodama A, Bauer S, Komatsu A, Asoh H, Ono S, Schmuki P. Bioactivation of titanium surfaces using coatings of $\mathrm{TiO} 2$ nanotubes rapidly pre-loaded with synthetic hydroxyapatite. Acta Biomaterialia 2009;5:2322-2330.

20. Raja KS, Misra M, Paramguru K. Deposition of calcium phosphate coating on nanotubular anodized titanium. Mater Lett 2005;59: 2137-2141.

21. Ishizawa $H$, Fujino $M$, Ogino $M$. Mechanical and histological investigation of hydrothermally treated and untreated anodic titanium oxide films containing $\mathrm{Ca}$ and P. J Biomed Mater Res 1995; 29:1459-1468.

22. Bellows CG, Aubin JE, Heersche JNM. Initiation and progression of mineralization of bone nodules formed in vitro: The role of alkaline phosphatase and organic phosphate. Bone Mineral 1991; 14:27-40.

23. Davis JE. Mechanisms of endosseous integration. Int J Prosthodontics 1998;11:391-401.

24. Schwartz Z, Lohmann $\mathrm{CH}$, Oefinger J, Bonewald LF, Dean DD, Boyan BD. Implant surface characteristics modulate differentiation behavior of cells in the osteoblastic lineage. Adv Dental Res 1999; 13:38-48.

25. Tambasco de Oliveira $P$, Nanci A. Nanotexturing of titaniumbased surfaces upregulates expression of bone sialoprotein and osteopontin by cultured osteogenic cells. Biomaterials 2004;25: 403-413.

26. Gu YX, Du J, Zhao JM, Si MS, Mo JJ, Lai HC. Characterization and preosteoblastic behavior of hydroxyapatite-deposited nanotube surface of titanium prepared by anodization coupled with alternative immersion method. J Biomed Mater Res B Appl Biomater 2012;100:2122-2130.

27. Chug A, Shukla S, Mahesh L, Jadwani S. OsseointegrationMolecular events at the bone-implant interface: A review. J Oral Maxillofacial Surg Med Pathol 2013;25:1-4.

28. Ma Q, Li M, Hu Z, Chen Q, Hu W. Enhancement of the bioactivity of titanium oxide nanotubes by precalcification. Mater Lett 2008; 62(17-18):3035-3038.

29. Surmenev RA, Surmeneva MA, Ivanova AA. Significance of calcium phosphate coatings for the enhancement of new bone osteogenesis-A review. Acta Biomaterialia 2014;10:557-579.

30. Svanborg LM, Hoffman M, Andersson M, Currie F, Kjellin P, Wennerberg $A$. The effect of hydroxyapatite nanocrystals on early bone formation surrounding dental implants. Int J Oral Maxillofacial Surg 2011;40:308-315.

31. De Ranieri A, Virdi AS, Kuroda S, Shott S, Leven RM, Hallab NJ, Sumner DR. Local application of rhTGF- $\beta 2$ enhances peri-implant bone volume and bone-implant contact in a rat model. Bone 2005;37:55-62.

32. Diefenbeck M, Mückley T, Schrader C, Schmidt J, Zankovych S, Bossert J, Jandt KD, Faucon M, Finger U. The effect of plasma chemical oxidation of titanium alloy on bone-implant contact in rats. Biomaterials 2011;32:8041-8047.

33. Karlsson J, Jimbo R, Fathali HM, Schwartz-Filho HO, Hayashi M, Halvarsson M, Wennerberg A, Andersson M. In vivo biomechanical stability of osseointegrating mesoporous TiO2 implants. Acta Biomaterialia 2012;8:4438-4446.

34. Marin C, Granato R, Suzuki M, Gil JN, Piattelli A, Coelho PG. Removal torque and histomorphometric evaluation of bioceramic grit-blasted/acid-etched and dual acid-etched implant surfaces: An experimental study in dogs. J Periodontology 2008;79:1942-1949.

35. Hägi TT, Enggist L, Michel D, Ferguson SJ, Liu Y, Hunziker EB. Mechanical insertion properties of calcium-phosphate implant coatings. Clinical Oral Implants Res 2010;21:1214-1222.

36. Le Guéhennec L, Soueidan A, Layrolle P, Amouriq Y. Surface treatments of titanium dental implants for rapid osseointegration. Dental Mater 2007;23:844-854.

37. Ueda K, Kawasaki Y, Narushima T, Goto T, Kurihara J, Nakagawa $\mathrm{H}$, Kawamura $\mathrm{H}$, Taira M. Calcium phosphate films with/without heat treatments fabricated using RF magnetron sputtering. J Biomech Sci Eng 2009;4:392-403.

38. Pittrof A, Bauer S, Schmuki P. Micropatterned TiO2 nanotube surfaces for site-selective nucleation of hydroxyapatite from simulated body fluid. Acta Biomaterialia 2011;7:424-431.

39. Sul Y-T. The significance of the surface properties of oxidized titanium to the bone response: Special emphasis on potential biochemical bonding of oxidized titanium implant. Biomaterials 2003;24:3893-3907.

40. Park J-W, Kim Y-J, Jang J-H, Kwon T-G, Bae Y-C, Suh J-Y. Effects of phosphoric acid treatment of titanium surfaces on surface properties, osteoblast response and removal of torque forces. Acta Biomaterialia 2010;6:1661-1670. 